\title{
Research of Grading System of Real-time Detection for Head of Garlic Based on Machine Vision
}

\author{
Liqing Zhao ${ }^{1,2, a *}$, Wei Song ${ }^{1, b}$ \\ ${ }^{1}$ Department of Electromechanical Engineering, Qingdao Agricultural University, Qingdao 266109, \\ China \\ ${ }^{2}$ Department of Engineering, Shenyang Agricultural University, Shenyang 110866, China \\ azhlq017214@163.com, bsongwei872119300@126.com \\ * Corresponding Author: zhlq017214@163.com
}

Keywords: Head of garlic; Vision by machine; Real-time detection; Grading

\begin{abstract}
This paper studies a kind of grading system of real-time detection for head of garlic based on machine vision. The system, which consists of transportation system of head of garlic, vision recognition system, single-chip control system and grading operation system, can be used to detect the size and shape of head of garlic. During the period of real-time detection and grading, the head of garlic is transported with single-row form. The real-time detection and grading shall be completed by intelligently recognizing with vision recognition system, responding to the recognition signal using single chip, issuing an order to the grading operation system and correspondent grading actions made by the operation system.
\end{abstract}

\section{Introduction}

At present, the computer grading technology for fruits has matured at home and abroad. However, there are still a few researches about the computer grading technology for the head of garlic at home. The grading of head of garlic is the most important process which can make an access for market circulation, which shall directly affect the packing, transportation and effectiveness and profits of sales. In order to improve the quality of head of garlic, profits of garlic merchants and make the products more standard, the accurate grading for head of garlic shall be conducted. However, the accurate grading at home takes the manual grading and mechanical grading as the main forms. The manual grading form needs huge labor intensity and the subjective assessment shall easily be subject to the personal vision, mood and tiredness, therefore boosts poor accuracy. As a result, the grading can't be conducted fully according to the grading standard. On the other hand, it is easy for mechanical grading to cause the garlic to suffer from the mechanical damage, which shall affect the quality of head of garlic ${ }^{[1-8]}$. This paper proposes a kind of grading system of real-time detection for head of garlic based on machine vision. This system Safety Technology Norm of Exporting for Garlic in Shandong Province $3^{[9]}$ (hereinafter refers to the Norm 3) requires that the system shall be able to detect as well as grade the head of garlic in real-time. The system gets rid of the shortcomings of manual and mechanical grading, thus can improve the labor productivity and promote the market competitiveness.

\section{Operating Principle of System}

In the operation of the grading system of real-time detection for head of garlic based on machine vision, the head of garlic is transported forward by using the transportation system with a constant speed of $5 \mathrm{~m} / \mathrm{s}$ and single row. When the head of garlic approaches the proximity switch of vision detection and recognition system of the machine, the proximity switch is able to detect the head of garlic fast, effectively and accurately and trigger the vision detection and recognition system of the

This work was supported by the Natural Science Foundation of Shandong Province (ZR2011FM034) 
machine. The vision system of machine can extract the information about the size and shape of head of garlic from the transported garlic with fast speed as well as can send the processing signal to the single-chip control module; after the single-chip control module receives the signals from the vision system of machine, it shall send the order of implementing the grading actions to the grading system. The grading system controls the complementing system to conduct the actions, thereby realizes the grading of head of garlic.

The grading system of real-time detection for head of garlic based on machine vision consist of transportation system for head of garlic with single row, machine vision detection and recognition system, single-chip control system and grading system. They are shown as follows in Fig 1. This system conducts the grading action for head of garlic with the productivity approaching to $4 \sim 7 \mathrm{t} / \mathrm{h}$ according to requirements in Norm 3.

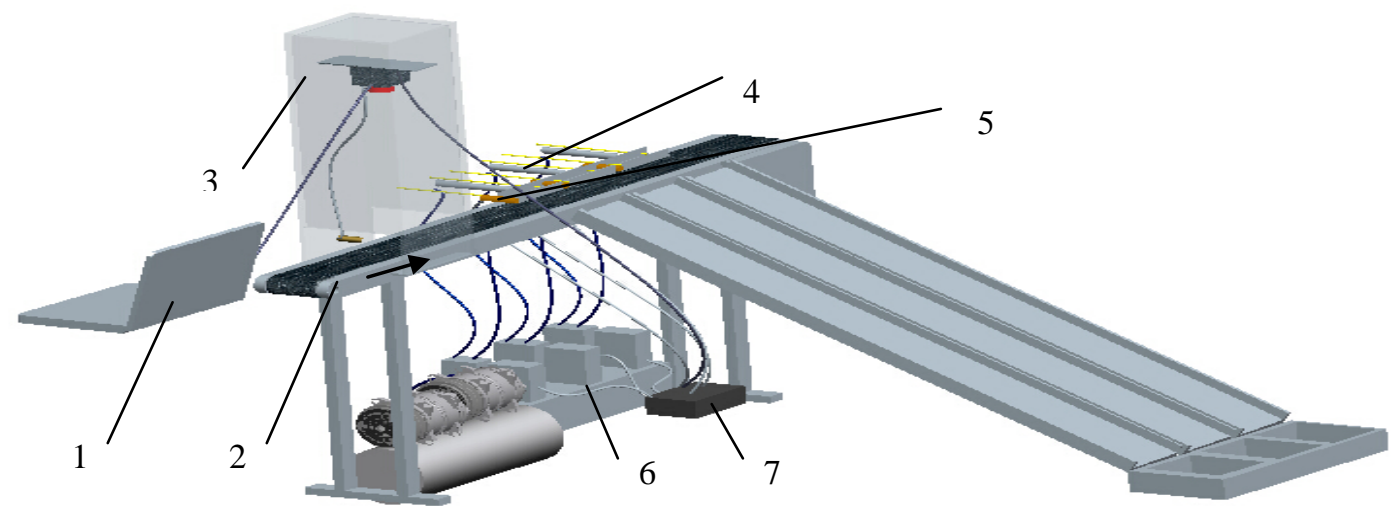

1. Dynamic detection and processing system of computer 2. Transportation system for head of garlic with single row 3. Machine vision detection and recognition system 4. Control module 5.Grading system 6. Grading actuator 7. Proximity switch (photoelectric sensor)

Fig.1 operating principle of system

\section{Design of system hardware}

\section{Vision detection and recognition system}

The vision detection and recognition system shall consist of Omron FQ2 smart camera, darkroom, proximity switch and computer (as shown in the Fig 1), among which, the FQ2 smart camera comes with a lighting device. A darkroom environment can only be created and the black conveyors are used in order to make it is easy for the camera to recognize the head of garlic. There are two cables for the FQ2 smart camera: one is the FQ Ethernet cable which is used to connect the computer to conduct the initial setting for the camera and dynamic monitoring for the whole operating process; the other is IO cable which is used to supply power for smart camera and output the signal being processed by the smart camera. When the sample of head of garlic approaches to the proximity switch, the proximity approaches triggers the camera and the camera shall output the control system of grading signals. The whole process shall be monitored by the computer (as shown in Fig 2).

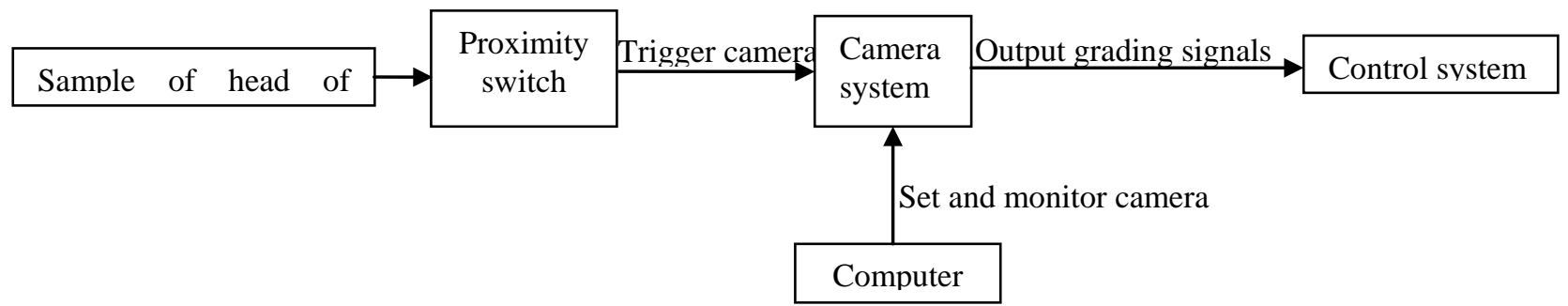

Fig.2 Process of vision detection and recognition 


\section{Vision detection and recognition system}

As shown in Fig 3, the control system mainly consists of STC89C52 single-chip microcomputer. The control system shall be used between the processes of vision detection and recognition system as well as the grading system. On one hand, it can be used to receive the result signal of vision detection and recognition system and on the other hand, it can be used to control the grading system's actions to achieve the sorting. The camera shall send the OUT0, OUT1 and OUT2 signals of its output-ends to the P00, P01 and P02 single-chip microcomputers. After the signals are processed by the single-chip microcomputers, the output-ends of P10, P11 and P12 shall control the grading system to respectively conduct the corresponding grading actions of Grade I, Grade II and Grade III.

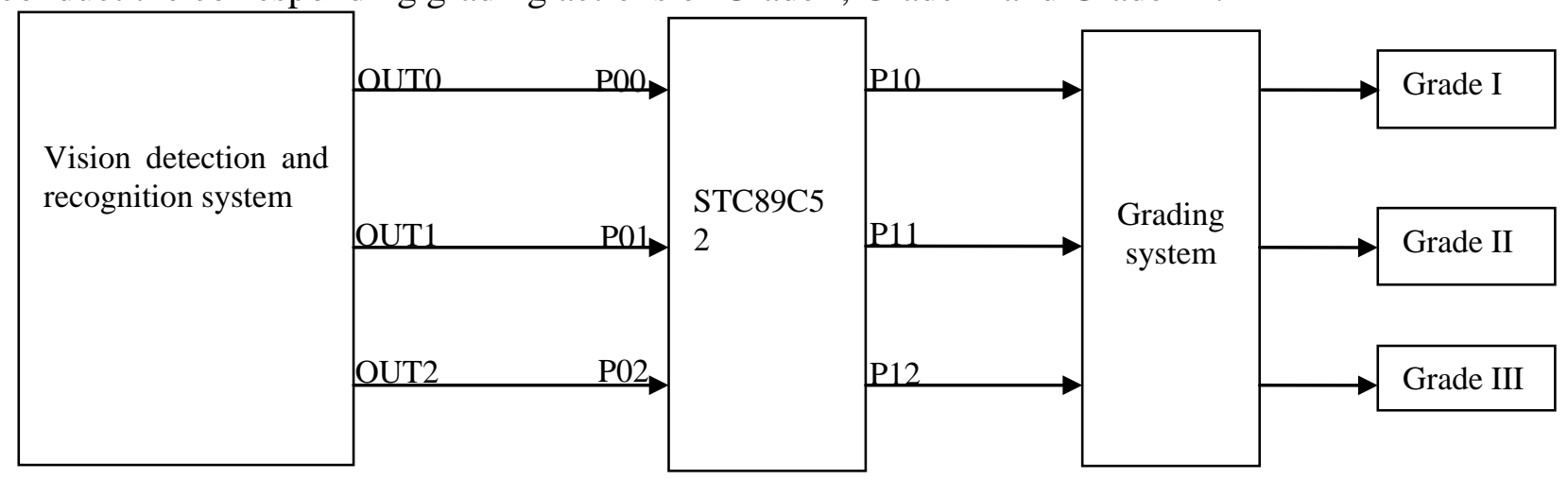

Fig.3 Control process of control system

\section{Grading system}

As shown in Fig 4, the grading system is mainly made up of OUTSTANDING 750-30L air compressor, YOLON three-position solenoid valve base, three pairs of YOLON three-position five-path solenoid valves and three pairs of AirTAC cylinders. Before grading, firstly start up the air compressor to get a certain pressure, and use it. The air compressor, solenoid valve base, solenoid valve and cylinder shall be connected with each other by using the high-quality $6 * 4 \mathrm{~mm}$ PU air tubes. The control system shall respectively control the No.1 solenoid valve, No.2 solenoid valve and NO.3 solenoid valve while the solenoid valve of grading system shall control the telescopic motion of cylinder to conduct the final grading for the head of garlic.

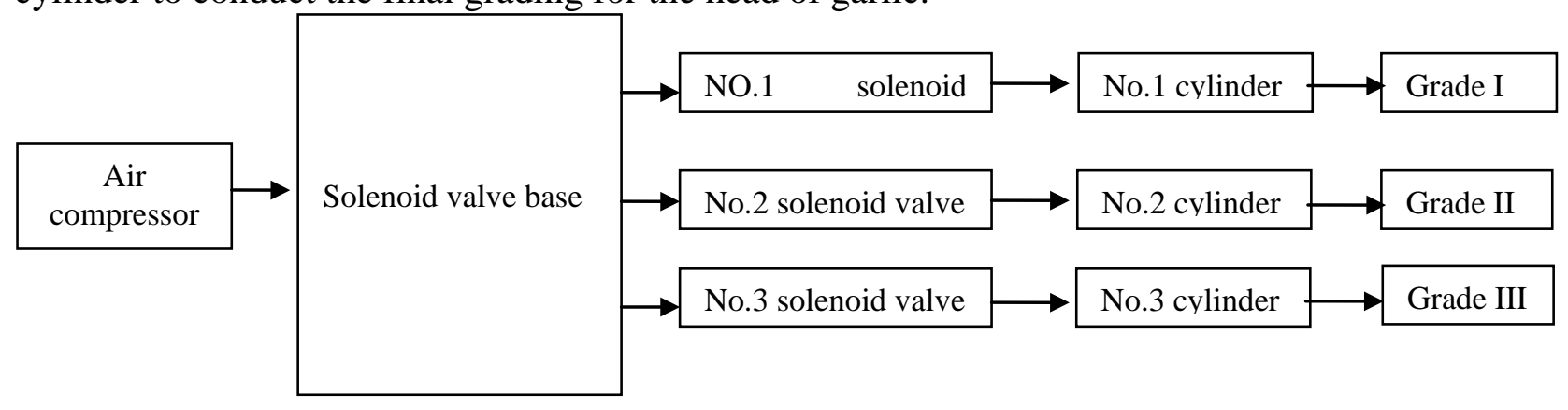

Fig.4 Control process of grading system

\section{Design of system software}

\section{Vision detection and recognition system software}

Firstly set the IP address and subnet mask of computer and use the special-use Ethernet cables to connect the computer and smart camera. Open the software TouchFinder for PC and set the size, shape, color and area of head of garlic (as shown in Fig 5). Adjust the shutter speed and focusing knob of camera to make the brightness of picture of garlic head more appropriate. Adjust the delaying time from inputting the triggering signals to inputting picture. After the setting, open the picture and conduct the dynamic monitoring for the state. 


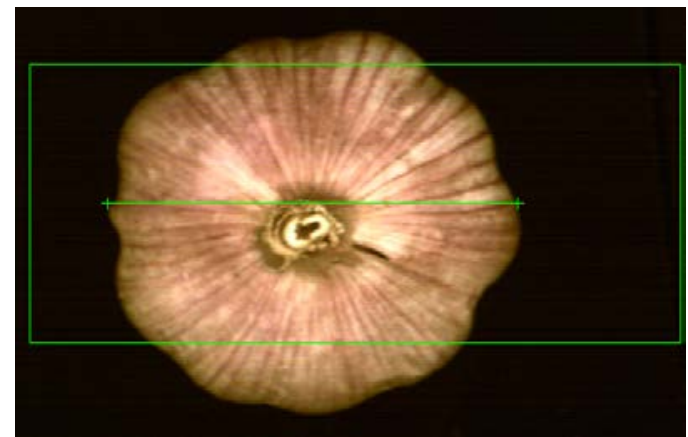

a. Detection of size

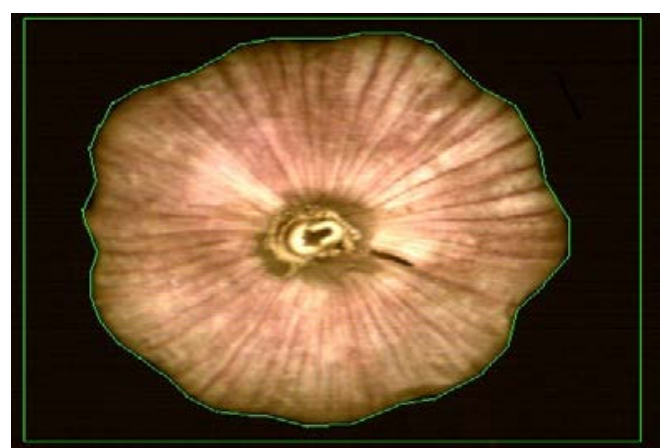

b.Detection of shape

Fig.5 Setting of vision software

\section{Software of control system}

The software of control system is mainly an external interrupt program of STC89C52 single-chip microcomputer. As shown in Fig 6, this program shall put the outputting signals of vision detection and recognition system into a queue and take out the signals of first address of the queue. After that, the signals stored in other address shall move an address towards the first address of queue in turn. As a result, the original second signal shall be stored at the first address and wait for being taken out to judge about the grades of garlic head.

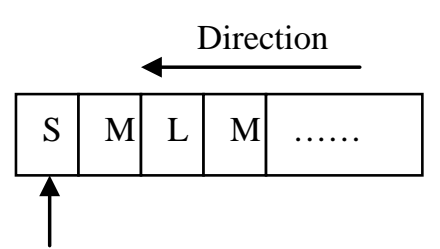

First address

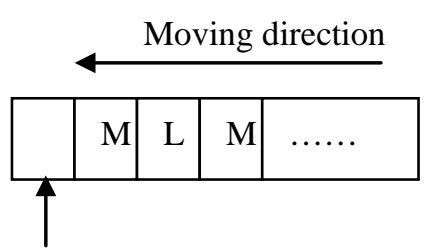

First address

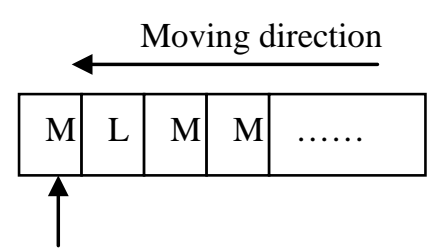

First address

Fig.6 Access modes of signals

\section{Summary}

According to Norm 3, the grading system of real-time detection for head of garlic based on machine vision shall conduct the fast-speed and real-time nondestructive detection grading for the size and shape of garlic heads and achieve the speed required during the real grading process, which improves the productivity and quality of garlic heads of China, what's more, enhances the market competitiveness of China's garlic. All those shall lay a foundation for the development of grading for garlic based on machine vision.

\section{References}

[1] Yang Songxia,Lu Huazhong,Lv Enli,Xu Jinfeng.Research Status on Spherical Fruits Mechanical Grader[J].Journal of Agricultural Mechanization Research,2012,08:235-239. (In Chinese with English abstract)

[2] Jyoti A K, Computer vision and image analysis based techniques for automatic characterization of fruits-a review [J].International Journal of Computer Applications, 2012, 50(6):6-12.

[3] Abbasgholipour M. Color image segmentation with genetic algorithm in a raisin sorting system based on machine vision in variable conditions [J]. Expert Systems with Applications, 2011, 38(4): $3671-3678$. 
[4] Jiang Huanyu, et al. Real Time Intelligent Inspecting and Grading Line of Fruits[J]. Transactions of the Chinese Society of Agricultural Engineering, 2002, 06:158-160. (In Chinese with English abstract).

[5] Chen Yanjun, Zhang Junxiong, Li Wei, et al. Grading method of apple by maximum cross-sectional diameter based on computer vision[J]. Transactions of the CSAE, 2012, 28(2): 284288. (In Chinese with English abstract)

[6] Chong V K,Kondo N,Ninomiya K, et al.Features extraction for eggplant fruit grading system using machine visioin[J].Applied Engineering Agriculture,2008,24(5):675-684.

[7] Li Kai, Yang Yanli, Liu Kai, et al. Determination and grading of Anthurium based on machine vision [J]. Transactions of the Chinese Society of Agricultural Engineering (Transactions of the CSAE), 2013, 29(24): 196-203. (In Chinese with English abstract)

[8] Wang Runtao, Zhang Changli, Fang Junlong. et al. Soybean seeds selection based on computer vision[J]. Transactions of the CSAE, 2011, 27(8): 355-359. (In Chinese with English abstract)

[9] DB37/T661.3-2007, Safety Technology Norm of Exporting for Garlic in Shandong Province $3[\mathrm{~S}]$. 